UCRL-JC-135867

PREPRINT

\title{
X-Point Heating, Potentials, and Temperature Asymmetries in Edge Plasmas
}

\author{
Ronald H. Cohen \\ Xueqiao Xu \\ Michael J. Schaeffer
}

This paper was prepared for submittal to Intl. Workshop on Plasma Edge Theory

Tajimi, Japan,

October 4-6, 1999

October 1, 1999

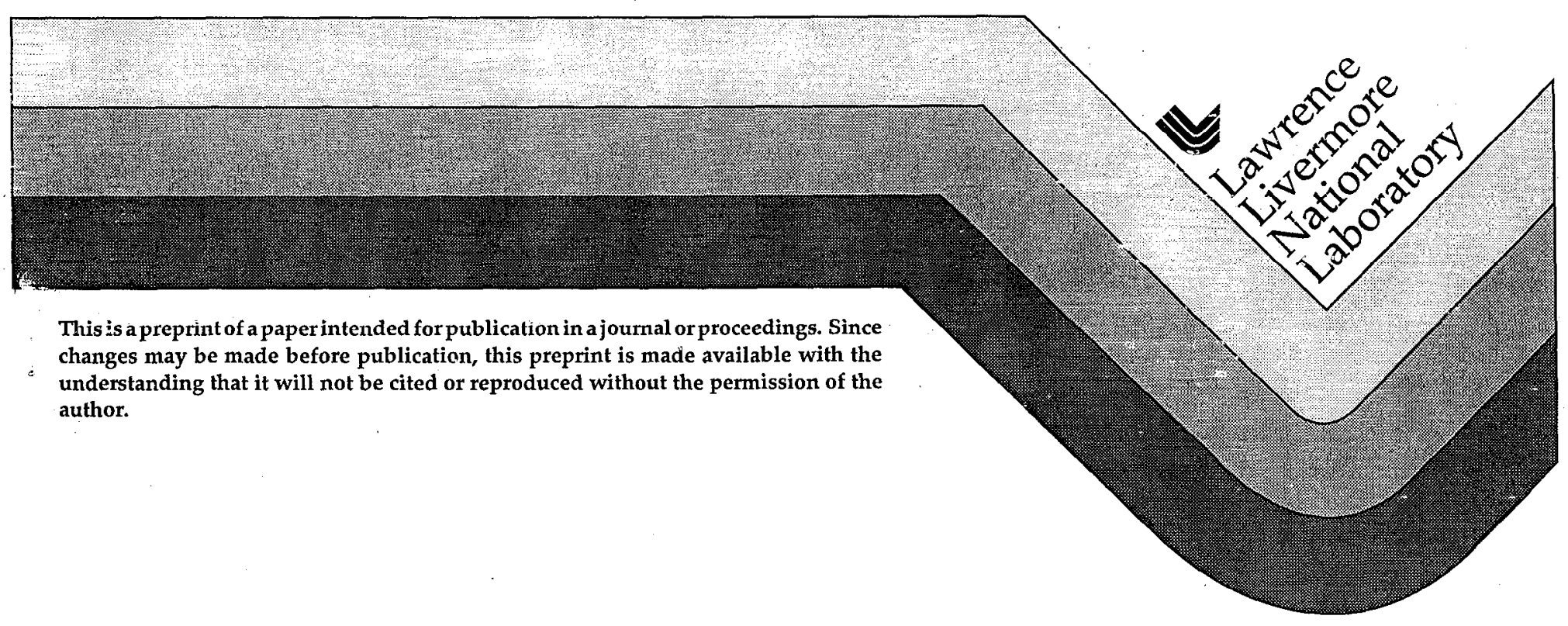




\section{DISCLAIMER}

This document was prepared as an account of work sponsored by an agency of the United States Government. Neither the United States Government nor the University of California nor any of their employees, makes any warranty, express or implied, or assumes any legal liability or responsibility for the accuracy, completeness, or usefulness of any information, apparatus, product, or process disclosed, or represents that its use would not infringe privately owned rights. Reference herein to any specific commercial product, process, or service by trade name, trademark, manufacturer, or otherwise, does not necessarily constitute or imply its endorsement, recommendation, or favoring by the United States Government or the University of California. The views and opinions of authors expressed herein do not necessarily state or reflect those of the United States Government or the University of California, and shall not be used for advertising or product endorsement purposes. 
Contrib. Plasma Phys. vol (year) num, $\mathrm{p}-\mathrm{p}$

\title{
X-Point heating, potentials, and temperature asym- metries in Edge Plasmas
}

\author{
Ronald H. Cohen ${ }^{a}$, Xueqiao Xu ${ }^{a}$, Michael J. Schaeffer ${ }^{b}$ \\ aLawrence Livermore National Laboratory, Livermore, CA 94550, USA \\ ${ }^{b}$ General Atomics, San Diego, CA 92186-5609, USA \\ e-mail:rcohen@llnl.gov
}

\begin{abstract}
Three-dimensional simulations of turbulence in the boundary plasma of a diverted tokamak indicate strongly localized heating and radial currents on closed flux surfaces in the vicinity of the $\mathrm{X}$ point, and oversil hurstiness. We apply a long-mean-free path theory of particle and energy exchange between passing and potential-trapped electrons to show that these turbulent phenomena combined with natural fueling asymmetries may lead to electron temperature, density and potential maxima in this region. Such maxima have been observed on the DIII-D tokamak.
\end{abstract}

\section{INTRODUCTION}

The edge region of a tokamak is characterized by large fluctuations in density, temperature and electrostatic potential. These fluctuations are generally believed to be responsible for the cross-field particle and heat transport in this region, and thus have a major impact on the width of the SOL and on fueling of the core plasma from particles ionized in the SOL, and regulation of these fluctuations is thought to be a key ingredient in the transition to enhanced confinement $(\mathrm{H})$ modes.

We note here that turbulence can also injpact the detailed structure of the plasma. equilibrium; in particular, for a diverted tokamak, concentration of turbulently driven, bursty, radial currents and Ohmic heating in the vicinity of the $\mathrm{X}$ point can contribute to formation of density, potential and temperature maxima in that region. This is in addition to peaking that results naturally from the strong concentration of particlo fueling near the $\mathrm{X}$ point because of its proximity to neutral gas sources.

The physical picture for the turbulence-produced asymmetries is closely linked to the physics of the resistive $X$-point mode, a recently discovered instability, described elsewhere at this meeting [1], which may be responsible for much of the turbulenoe in the edge of diverted tolkamaks under L-mode conditions.

Because the electron mean free path $L_{m f p}$ is comparable to the length of the potential maximum, we calculate the temperature and potential differences resulting from the turbulent sources in both short and long mean-free-path limits. Perhaps surprisingly, the latter approximation can produce stronger asymmetries for the parameters of interest, because of the limited rate of energy exchange between electrons trapped in the potential well near the $X$ point and those passing to the rest of the flux surface [2], [3].

This paper is organized as follows. In Sec. 2 we describe the relevant simulation results and experimental data. In Sec. 3 we review the theory of weakly collisional energy exchange and calculate expected temperature and potential differences and their dependence on applied power, turbulently induced radial current, density asymmetry, 


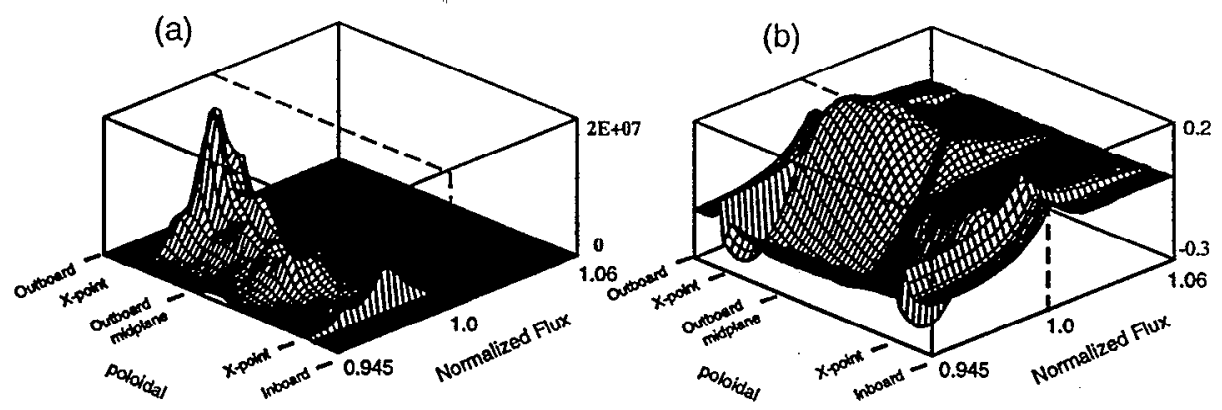

Fig. 1: BOUT simulations of edge turbulence for uniform equilibrium density and temperature on a flux surface. (a) instantaneous resistive heating rate averaged over toroidal angle; (b) instantaneous radial current averaged over toroidal angle.

and upstream electron temperature. We also briefly review results of a fully collisional calculation. Section 4 is a discussion, including..remarks about other edge-plasma applications of the theory discussed here.

\section{SIMULATION RESULTS AND EXPERIMENTAL DATA}

The BOUT code solves 3-dimensional nonlincar fluid cquations for electron and ion parallel velocities and temperatures $T_{e}$ and $T_{i}$, density $n$, electrostatic potential $\Phi$, and the parallel component of the vector potential, taking into account the presence of closed and open field lines in a realistic geometry that includes X-point effects, ungnelic shear, and sheath boundary conditions.

A series of simulations were performed to follow the transition from low (L) to high (I) confinement modes. A power source is provided along the inner strip of the simulation domain, to mimic the effect of power transported from the core plasma.. As this power is increased, a transition, interpreted as the.L-H transition, is observed, from a state of high turbulent fluxes to a state with lower fluxes and higher rotational shear. These runs and their connection to the L-H transition will be discussed more ., fully in a separate publication [4].

In the L-mode phase, the turbulence is broadly distributed in poloidal angle, peaks somewhat outboard of the X point and drops closer to it. "Two striking features are that fluctuating parallel currents produce a strong - as much as $1-2 \mathrm{MW} / \mathrm{m}^{3}-$ localized heating near the $\mathrm{X}$ point (fig. la) and that the vorticity fluctuations lead to substantial radial current near the $X$ point. The strength of the latter is indicated in fig. $1 \mathrm{~b}$, where the resultant potential is shown for a case with an assigned uniform density profile and calculated uniform temperature profile; a potential difference of order $0.3 T_{e}$ is driven. Another important feature of the simulations is that the turbulence, and the driven potential, are bursty in time.

The origin of these effects is the magnetic shear in the neighborhood of the $X$ point, which has the effect of raising both the radial and poloidal components of the wavenumber of a mode in this region.

The radial current from the geodesic $(\mathbf{B} \times \nabla \Psi$, where $\Psi$ is the poloidal flux $)$ component of the summed electron and ion momentum fluid equations has a turbulently 
driven component:

$$
\left\langle J_{\Psi}\right\rangle \approx \frac{M_{i} c}{\mathcal{J}|\nabla \Psi|} \frac{\partial}{\partial \Psi}\left\{\left\langle\left(\mathrm{v}_{E}+v_{\|_{i}} \mathrm{~b}\right) n R v_{g}\right\rangle \cdot \nabla \Psi \mathcal{J}\right\}
$$

where $v_{E}$ is the $\mathbf{E} \times \mathbf{B}$ velocity, $v_{g}$ is the total geodesic drift velocity (including the diamagnetic contribution), and $\mathcal{J}$ is the Jacobian. The averaging indicated by \langle\rangle is over the fluctuation timescale and toroidal angle. This Reynolds-stress-like term varies as $k_{r}^{2} k_{g}|\tilde{\Phi}|^{2}$, where $\tilde{\Phi}$ is the fluctuating potential, and so has a strong peaking tendency which can more than compensate for the falloff of $\tilde{\Phi}$. This radial current is closed by a parallel electron current.

The instantaneous parallel current $J_{\|} \propto \partial \tilde{\Phi} / \partial s$ also peaks near the x point, because of a transition of the mode from electromagnetic to electrostatic as the $\mathrm{x}$ point is approached: this transition occurs [5] when the parameter $\left.\left(k_{1}^{2} c^{2} / \omega_{p e}^{2}\right)\left(1+i \nu_{s}\right) / \Omega\right)$ becomes large; here $\Omega$ is the wave frequency Doppler shifted by $\mathbf{E} \times \mathbf{B}$ rotation. This transition allows the mode to drop its amplitude rapidly in that region (and is what enables large growth rates for the resistive X-point mode [1]).

Experimentally, probe and Thompson-scattering data from low-n, low- $T$ DIII-D shots indicate that, on closed flux surfaces within a $\mathrm{cm}$. or so of the separatrix, there are local maxima in density $\left(1.2-2 \times 10^{1} 3 \mathrm{~cm}^{-3}\right.$ near the $\mathrm{X}$ point vs. $0.8 \mathrm{~cm}^{-3}$ upstream), electrostatic potential $(40-150 \mathrm{~V}$ near the $\mathrm{X}$ point vs. $10-30 \mathrm{~V}$ upstream) and electron temperature $(50-80 \mathrm{~V}$ near the $\mathrm{X}$ point vs. $35 \mathrm{~V}$ upstream). The data indicates significant variability (within the ranges indicated) in the $\mathrm{X}$-point quantities during the course of a shot.

\section{Heating and Potential Models}

$\therefore$ In this section we address whether there might be a connection between localized turbulence effects and the experimentally observed asymmetries presented in the preceding section. Due to space limits, we only briefly summarize conclusions for the familiar collisional limit and then consider the long-mean-free-path regime.

The average particle source is localized near the $X$-point region because of proximity to neutral-gas sources. The pulses of turbulently driver current can introduce transient increments to this source. Because of the limited ion parallel transport and turbulent plus convective losses upstream, a significant density peak can be maintained near. the $\mathrm{X}$ point. If, due to high electron parallel thermal conductivity; we approximate $T_{e} \approx$ const, then electron parallel momentum equation yields $\Delta \phi \approx T_{e} \ln n_{\max } / n_{\min }$ roughly consistent with the DIII-D measurements. (The same estimate follows also in. the collisionless limit). The parallel electron current that responds to the (transient) radial current can be added to this as $\int d s \eta J_{\|}$where $\eta$ is the resistivity. The steadystate temperature difference that follows from parallel electron heat conduction is small, only a few percent even for $\sim 1 \mathrm{MW}$ of power applied in a $\sim 5 \mathrm{~cm}$ wide zone near the $\mathrm{x}$ point for the parameters of the DIII-D study. Transient responses to bursts in the turbulence could be appreciable if the bursts are sufficiently rare, but then the probability of experimental observance becomes small.

The electron mean free path $L_{m f p}$ can be comparable to the extent of the region where the turbulent heating and current transport occur, especially for low-density or high temperature edge plasmas. Hence one is led to consider the limit of long $L_{m f p}$. There are theoretical and computational studies [2], [3] which develop a theory of 
collisional particle and energy exchange between electrostatically trapped and passing. electrons and provide the basis for calculation of potentials and temperature differences. The essential results are equations for the rate of change of trapped density $n_{t r}$ and energy $n_{t r} E t r$ for a specified confining potential $\Delta \Phi$ and magnetic mirror ratio $R$. For brevity, we display only the expressions for $R \geq 1$ and in our applications neglect mirroring effects $(R \approx 1)$. Then we have:

$$
\frac{d n_{t r}}{d t}=-G+S \quad, \quad \frac{d}{d t}\left(n_{t r} E_{t r}\right)=-Q+(n T)_{v}+P
$$

where $S$ and $P$ are respectively the volumetric particle and energy sources, while $G$ and $Q$, the particle and energy fluxes across the velocity-space boundary dividing passing and trapped electrons are given by:

$$
G=\hat{G}\left(1-\frac{n_{m}}{n_{t}} \lambda^{1 / 2} e^{\phi}\right) \quad, \quad \frac{Q-\Delta \Phi G}{\hat{Q}-\Delta \Phi \hat{G}}=1-\frac{n_{m}}{n_{t}} \lambda^{-1 / 2} e^{\phi}
$$

Here $\lambda=T_{t} / T_{m}, n_{m}$ and $T_{m}$ are the density of untrapped electrons at the potential well edge, which we can take as. the density at the tokamak midplane, $\phi=e \Delta \Phi / T_{t}, n_{t}$ and $T_{t}$ are the density and temperature of the Maxwellian that the trapped distribution approaches at low energy, and $\hat{G}(\Delta \Phi, R)$ and $\hat{Q}(\Delta \Phi, R) \approx\left(e \Delta \Phi+T_{t} \hat{G}\right)$ are the particle and energy fluxes in the absence of passing particles. The relationship between $n_{t r}$ and $n_{t}$ is spelled out in Ref. [3]. Many papers have derived expressions for $\hat{G}$, starting with Pastukhov's [7] and continuing through an elegant expansion treatment by Khudik [8]. All have the form $\hat{G}=(n / \tau)[I(\phi) / G(R, \phi)] e^{-\phi}$ where for large $\phi$ and $R, I \sim 1 / \phi$ and $G \sim \ln R$. We use here the form of Najmabadi et al., [9], as it works well even for $\phi \sim 1$; but we approximate to Najmabadi et als $w$ by $w=(1+1 / R)^{1 / 2}$ so that $\hat{G}$ approaches a sensible limit as $\phi \rightarrow 0$ but is quite close to the full expression for $\phi \geq 1$. [There'is a missprint in Ref. [9]; Eq. (39b) for $w$ is correct, not the expression following Eq. (42).]

We first consider steady-state solutions of Eqs. (2) with $S=0$ (since the steadystate source replenishes on the long ion confinement timescale). Plots of $\Delta \Phi$ and $\lambda=T_{t} / T_{m}$ are shown in Fig. 2; here, $n_{x}$ is the total density in the $\mathrm{x}$-point (potential well) region. Obtaining $\lambda$ significantly different from 1 requires a large density contrast and/or a $P \gtrsim 10 \mathrm{Wcm}^{-3}$, which is energetically feasible provided the heating volume is small, but an order of magnitude larger than observed in our sinulations. The results indicate that as $P$ is increased, $\Delta \Phi$ rises with, but not as fast as, $T_{t}$. We note that in this weakly collisional regime, any heating source acting on the trapped electrons, even one not localized, produces some temperature asymmetry, because the the potential-trapped electrons are better confined.

Monte-Carlo studies [10] indicates that finite-mean-free-path corrections to the long-mean-free-path physics can increase $\left(T_{x} / T_{m}\right)-1$ by several times (and thus become larger than predicted by either long- or short-mean-free-path theory); this can be attributed to boundary-layer effects limiting contact between passing and trapped particles. Hence an arbitrary-collisionality steady-state description might be able to account for the temperature asymmetries in DIII-D.

Next we consider the solution appropriate for ion current driven radially into this region from bursts of turbulence. This current must be closed by an electron current along field lines into the $\mathrm{x}$-point region. In the weakly collisional theory this appears as 

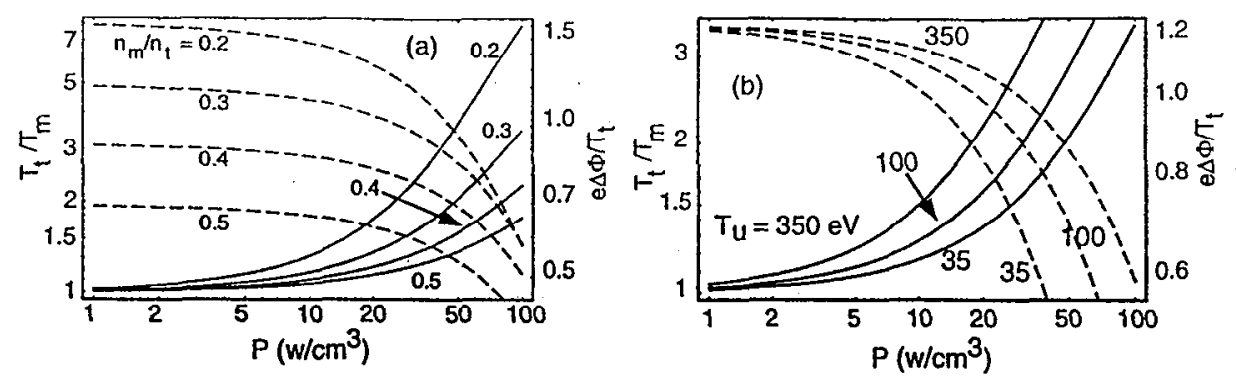

Fig. 2: Solutions of steady-state equations for temperature ratio (solid curves) and normalized potential difference (dashed curves). (a) results for several density ratios at fixed $T_{m}=35 \mathrm{eV}$; (b) results for several $T_{m}$ at fixed $n_{m} / n_{x}=0.3$.

a flux into the ilapped portion of electron velocity space. In terms of Eq. (2), this flux can be maintained by specifying a positive $d n_{t r} / d t$ or, equivalently, a negative source. (The latter could also describe loss due to electron radial transport). We show in Fig. 3 the potential difference and temperature ratio as a function of $\nu_{J} \equiv n^{-1} d n_{t r} / d t$ for a density ratio of $1 / 2$ and 1 .

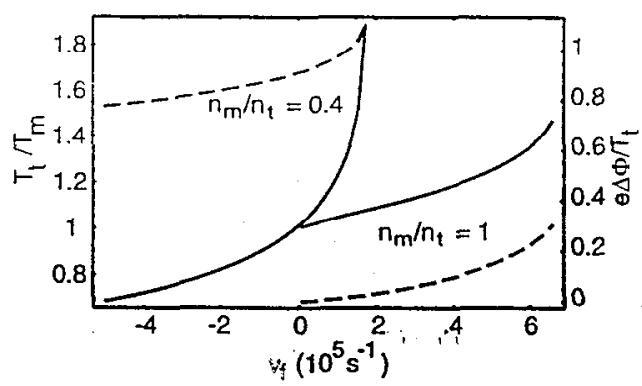

Fig. 3: Potential difference (dashed curve) and temperature ratio (solid curve) vs. electron return current parameter $\nu_{f}$.
For $n_{m} / n_{x}=1$, which applies also to the turbulence simulation of Fig. 2, $e \Delta \Phi \sim 0.3 T_{e}$ (the value from the turbulence simulation) can be obtained with $\nu_{J} \sim 2 \times 10^{18} \mathrm{~cm}^{-3} \mathrm{sec}^{-1}$. The temperature ratio is close to 1 . For $n_{m} / n_{x} \sim 1 / 2$, which is more typical of the DIII-D data, a lower $\nu_{I}, \sim 1 \times 10^{18} \mathrm{~cm}^{-3} \mathrm{sec}^{-1}$ can sustain $\Delta \Phi \sim T_{\varepsilon} / c$ and $T_{m} / T_{x} \sim 1.3$ as in the experiment. $P$ was $1 \mathrm{~W} \mathrm{~cm}^{-3}$ in these runs, but can also be set to zero with little change in the curves. The main power source is the energy gained by the passing electrons as they fall down their potential energy hill; as they become trapped and collide with previously trapped electrons, they raise $T_{t}$.

In the above example $\nu_{f}$ is about the ion collision frequency, i.e. small compared to the eleccron collision frequency, but still implies a density doubling time of order $10 \mu$ s (recall that ions are entering the volume radially, at the same rate.) Thus this process cannot be sustained. The long-time average of the turbulence-driven radial current must be zero. The simulations support this, with time intervals of inward and outward turbulence-driven (ion) current. The density ratio $n_{x} / n_{m}$ will fluctuate about a value grcater than 1 bccausc of the localization of the time-averaged density source. Then because potential confinement is nonlinear, the time-average of $T_{t} / T_{m}$ will be positive, as can be inferred from comparing the $\nu_{f}>0$ and $\nu_{f}<0$ portions of Fig. 3 .

\section{Discussion and conclusions}

The size and localization of turbulence-driven currents, the presence of localized heating from the turbulence, and the bursty nature of the turbulence malie an explanation 
for the observed temperature, density and potential asymmetries based on weakly colllisional processes plausible. Other mechanisms, including collisional impurity and ponderomotive effects, also merit exploration.

A consequence of the potential maximum is that, since it tends to extend into the scrape-off layer (SOL), it will influence the asymmetry of particle and power deposition on inboard and outboard divertor plates. The radial $\mathbf{E} \times \mathbf{B}$ advection in the divertor legs will be larger in the presence of the potential maximum than it would have been, and is oppositely directed in the main SOL and the divertor leg (where the SOL is in contact with relatively hot and cold plasmas, respectively, across the separatrix). The effects will of course change direction with that of the magnetic field.

Another application of the long-mean-free-path theory is to the SOL of spherical tokamaks. Because of the short connection length (poloidal field relatively large compared to toroidal field) and high mirror ratio (large variation in major radius along an SOL field line), $T_{e}$ may be rather high and $L_{m f p}$ may be of order of the connection length. In this case the potential well region is the main SOL, and the long- $L_{m f p}$ description of energy exchange, perhaps as modified by finite- $L_{m f p}$ corrections [10], would then provide the basis for expecting a significant temperature contrast between the main SOL and the divertor legs.

\section{Acknowledgements}

We thank D. D. Ryutov for stimulating dicussions. This work was performed for the U.S. Department of Energy under contracts W7405-ENG-48 at LLNL and DE-AC0398ER54463 at General Atomics.

\section{References}

[1] Myra, J.R., D'Ippoli'ro, D.A., Xu, X.Q., Cohen, R.H., Contrib. Plasma Phys., this issue

[2] Cohen, R.H., Bernstein, I.B., Dorning, J.J., Rowlands, G., Nucl. Fusion 20(1980) 1421

[3] Rensink, M.E., Cohen, R.H., Mirin, A.A., Tomasghke, G.P., Nucl. Fusion 24(1984) 49

[4] , XU, X.Q. invited paper, APS-DPP Conference, Seattle, WA, USA, Nov. 15-19, 1999, to be published in Phys. Plasmas.

[5] Berk, H.L., Cohen, R.H., Ryutov, D.D., Tsidulko, Yu.A., Xu, X.Q., Nucl. Fusion 33(1993)263

[6] Schaffer, M.J., Boedo, J., Moyer, R.A., Bull. Am. Phys. Soc. 43(1998)1889, Paper R8P5

[7] Pastukhov, V.P., Nucl. Fusion 14(1974)3

[8] KHudik, V.N., Nucl. Fusion bf $37(1997) 189$

[9] Najmabadi, F., Conn, R.W., Cohen, R.H., Nucl. Fusion 24(1984)75

[10] Rognlien, T.D., Bull. Am. Phys. Soc. 23(1978),754 Paper 1 Q5.

Received September 30, 1999. 